\title{
GCU
}

Glasgow Caledonian

University

University for the Common Good

\section{Exercise for osteoarthritis of the hip and knee}

Howe, Tracey

Published in:

Annual Review of Gerontology and Geriatrics

DOI:

10.1891/0198-8794.36.155

Publication date:

2016

Document Version

Author accepted manuscript

Link to publication in ResearchOnline

Citation for published version (Harvard):

Howe, T 2016, 'Exercise for osteoarthritis of the hip and knee', Annual Review of Gerontology and Geriatrics, vol. 36, pp. 155-168. https://doi.org/10.1891/0198-8794.36.155

\section{General rights}

Copyright and moral rights for the publications made accessible in the public portal are retained by the authors and/or other copyright owners and it is a condition of accessing publications that users recognise and abide by the legal requirements associated with these rights.

Take down policy

If you believe that this document breaches copyright please view our takedown policy at https://edshare.gcu.ac.uk/id/eprint/5179 for details of how to contact us. 


\section{CHAPTER 6}

Exercise for Osteoarthritis of the 5 Exercise for Osteoarthritis of the 6 Hip and Knee Hip and Knee

Tracey E. Howe

Osteoarthritis (OA) is the most common form of arthritis and is a painful, chron-

ically disabling joint disease that often significantly compromises function and independence. With the generalized rise in the age of the global population, the number of people suffering from musculoskeletal disorders throughout the world will increase dramatically over the coming decades. International clinical guidelines recommend exercise for the treatment of OA. Given the current and predicted future global burden of OA of the hip and knee, the aim of this chapter is to summarize the evidence from recent systematic reviews published in English on the effectiveness of land- and water-based exercise programs for people with $\mathrm{OA}$ of the hip and knee. The primary outcomes considered, based

AQ1 on the recommendations of the OMERACT III conference, are pain and function. Recent systematic reviews have examined the effectiveness of exercise on pain and function compared with controls: land- versus water-based exercise, individual versus group exercise, and exercise prior to surgery for people with OA of the hip, the knee, and lower limb (hip and knee combined). Generally, most forms of exercise have been demonstrated to have significant positive effects on pain and function at the end of the exercise program, but unless participants continue the program, the sustainability of these effects in the longer term are questionable. 
However, exercise appears to be a safe intervention, with few adverse effects reported. A number of considerations relating to the design and reporting of primary research in this area are discussed.

\section{INTRODUCTION}

Osteoarthritis (OA) is the most common form of arthritis and is a painful, chronically disabling joint disease that often significantly compromises function and independence. OA can present in the spine and hands, but the most frequently affected joints are the main load-bearing joints, the hips and knees. Approximately one in four people may develop symptomatic hip OA in their lifetime (Murphy et al., 2010); the lifetime prevalence for hip OA is estimated to be $25.3 \%$ but it is much higher for knee OA, 44.7\% (Murphy et al., 2008). The global age-standardized point prevalence for hip and knee OA in 2010 was 3.8\% (95\% uncertainty interval 3.6 to 4.1 ) and was higher in females peaking at age 50 years (March et al., 2014).

The symptoms of OA include pain, joint stiffness, muscle weakness and atrophy, altered proprioception, and limitations in functional activities and social participation. Joint malalignment may be present in progressive disease states and this can result in bone-on-bone joint pain. Hip and knee OA accounts for about $10.5 \%$ of the total disability burden in terms of years lived, with disabilities for all musculoskeletal diseases combined.

With the generalized rise in the age of the global population, the number of people suffering from musculoskeletal disorders throughout the world will increase dramatically over the coming decades. For example in the United States, the number of adults with arthritis is projected to increase from 47.8 million in 2005 to 67 million in 2030 (Hootman \& Helmick, 2006). This will result in an exponential increase in the global burden of disability from OA hip and knee; therefore, finding cost-effective and safe treatment to minimize the symptoms for people with OA hip and knee is imperative.

Traditionally, treatment of OA involves pharmacological interventions such as paracetamol/acetaminophen, nonsteroidal anti-inflammatory drugs, and intra-articular steroids. Nonpharmacological interventions include exercise, education, weight reduction, appliances, heat and cold therapy, transcutaneous electrical nerve stimulation, pulsed electromagnetic field, and laser and galvanic stimulation; joint replacement is the most common surgical intervention.

\section{THE ROLE OF PHYSICAL ACTIVITY AND EXERCISE}

It is well documented that regular moderate-intensity physical activity-such as 41 
benefits for general health of populations, such as reducing the risk of cardiovascular diseases, diabetes, colon and breast cancer, and depression; decreasing the risk of a hip or vertebral fracture; and helping control weight. Indeed, global recommendations on physical activity for health for adults are participation in moderate physical activity, for example, walking, cycling, and household chores for at least 30 minutes per session, five times per week, or vigorous activity for at least 25 minutes per session, three times per week (WHO, 2010a). It can be postulated then that participation in regular physical activity may be sufficient to protect against the development of OA or relieve symptoms of OA hip and knee. However, it has been reported that only $27.8 \%$ of individuals with arthritis engage in physical activity to a level that is sufficient to experience these benefits (Hootman, Macera, Ham, Helmick, \& Sniezek, 2003). The terms physical activity and exercise are often used interchangeably. Physical activity in daily life can be categorized into occupational, sports, conditioning, household, or other activities. Exercise is a subset of physical activity that is planned, structured, and repetitive and has the objective of improvement or maintenance of physical fitness or a desired therapeutic effect (Caspersen, Powell, \& Christenson, 1985). Exercise has been demonstrated to be effective in a number of groups, for example, improving balance in older people (Howe, Rochester, Neil, Skelton, \& Ballinger, 2011) and preventing and managing osteoporosis in postmenopausal women (Howe et al., 2011).

International clinical guidelines such as Osteoarthritis Research Society

AQ3 International (OARSI) guidelines for the nonsurgical management of knee OA (McAlindon et al., 2014; Roddy et al., 2006) recommend exercise as a low-cost safe intervention with few reported adverse effects. The aim of therapeutic exercise programs is to relieve the symptoms of OA of the hip or knee including pain, joint stiffness, muscle weakness, and subsequent reduction in function. Therapeutic exercise programs for OA hip and knee frequently include land- or water-based exercise designed to improve muscle strength, range of movement in the joint, or aerobic capacity, or combinations of these. These exercise programs are typically designed and supervised by health professionals but may also be undertaken at home as part of self-management strategies.

Outcome measurement in clinical studies requires the use of valid, reliable, and responsive measurement procedures that adequately capture important aspects of the condition. In 1997, participants at the OMERACT III conference agreed $(\geq 90 \%)$ on a core set of four domains for outcome measurement in future Phase III clinical studies of hip, knee, and hand OA. The four domains identified were pain, physical function, patient global assessment, and, for studies of at least 1 year, joint imaging (Bellamy et al., 1997). This has been further confirmed by Paskins, Sanders, and Hassell (2014) in their systematic review comparing 
patient experiences of the OA consultation with general practitioner (GP) attitudes and beliefs about $\mathrm{OA}$. The authors found that pain management and fear of disability were consultation priorities for patients. Patients seem keen to explore nonpharmacological interventions, whereas GPs reported frustration and lack of knowledge about issues to do with lifestyle changes.

Functioning and disability, according to the International Classification of Functioning, Disability and Health (ICF) (WHO, 2010b), are viewed as a complex interaction between the health condition of the individual and the contextual factors of the environment as well as personal factors. The ICF focuses on impact, creating a foundation and a common framework allowing all conditions to be compared using a common metric - the impact on the functioning of the individual "in his or her world."

Recognizing the global burden of OA of the hip and knee, this chapter summarizes the evidence from recent systematic reviews on the effectiveness of exercise programs for $\mathrm{OA}$ of the hip and knee on pain and function.

\section{Exercise and OA of the Hip}

Three systematic reviews have examined the effects of exercise on $O A$ of the hip (Fransen, McConnell, Hernandez-Molina, \& Reichenbach, 2014; HernándezMolina, Reichenbach, Zhang, Lavalley, \& Felson, 2008; Romeo, Parazza, Boschi, Nava, \& Vanti, 2013).

A recently updated Cochrane review, search updated to Feb 2013, (Fransen et al., 2014) included 10 studies (549 participants), 7 of which were judged as having a low risk of bias. The studies included participants with mostly mildto-moderate symptomatic hip OA, alone or with knee OA. Except for one study where participants enrolled in a tai chi program, all other participants underwent land-based exercise programs consisting of traditional muscle strengthening, functional training, and aerobic fitness programs, supervised either individually or as part of a group. Participants in the control groups did not exercise. Meta-analysis of high-quality evidence from nine studies (549 participants) demonstrated that exercise reduced pain (standardized mean difference [SMD] -0.38, 95\% CI -0.55 to -0.20 ) equivalent to 8 points on a scale $0-100$ (95\% CI 4 to 11 points; number needed to treat for an additional beneficial outcome $[$ NNTB] $=6$ ). Physical function also improved (SMD $-0.38,95 \% \mathrm{CI}-0.54$ to -0.05 ) equivalent to 7 points on a $0-100$ scale $(95 \%$ CI 1 to 12 points; NNTB $=6)$. Only three small studies (183 participants) evaluated quality of life and they demonstrated no benefit from exercise for participants' quality of life (SMD $-0.07,95 \%$ CI -0.23 to -0.36).

These findings are consistent with a previous high-quality meta-analysis of nine studies (1,234 participants) comparing exercise programs of durations lon41 
beneficial effect of exercise on pain with an effect size (ES) of -0.38 (95\% CI -0.68 to $-0.08 ; p=0.01)$, but there was high heterogeneity $\left(\mathrm{I}^{2}=75 \%\right)$ among studies. The heterogeneity was caused by one trial consisting of an exercise intervention that was not administered in person. When the authors removed this study leaving eight studies (493 participants) with a similar exercise strategy (specialized hands-on exercise training, all of which included at least some element of muscle strengthening), exercise demonstrated a benefit on pain with an ES of -0.46 ( $95 \%$ CI -0.64 to $-0.28 ; p<0.0001)$.

In contrast, a descriptive review involving 10 Studies, 2 of which involved manual therapy (Romeo et al., 2013), concluded that at mid- and long-term follow-up land-based exercises showed insufficient evidence of effectiveness with respect to pain and quality of life, but positive results were found for physical function. The authors also concluded that water-based exercises significantly reduced fall risk when combined with functional exercises. They stated that programs containing progressive and gradual exposure to difficult activities, education, and exercises promoted better outcomes, higher adherence to home program, and increased amount of physical activity, especially walking.

\section{Exercise and OA of the Knee}

Eight reviews examined studies investigating the effects of exercise on OA of the knee (Devos-Comby, Cronan, \& Roesch, 2006; Focht, 2006; Fransen et al., 2015; Jansen, Viechtbauer, Lenssen, Hendriks, \& de Bie, 2011; Juhl, Christensen, Roos, Zhang, \& Lund, 2014; Petrella, 2000; Roddy, Zhang, \& Doherty, 2005; Tanaka, Ozawa, Kito, \& Moriyama, 2013).

A recently updated Cochrane review (Fransen et al., 2015), search updated to May 2013, undertook meta-analysis of data immediately after completion of exercise programs, compared with controls. They defined exercise as any landbased nonperioperative therapeutic exercise regimen aimed at relieving the symptoms of OA, regardless of content, duration, frequency, or intensity. Highquality evidence from 44 studies (3,537 participants) indicated that pain was reduced (SMD $-0.49,95 \% \mathrm{CI}-0.39$ to -0.59 ), equivalent to 12 points (95\% CI 10 to 15 points) on a scale of $0-100$ points. Moderate-quality evidence from 44 studies (3,913 participants) showed that exercise improved physical function (SMD $-0.52,95 \%$ CI -0.39 to -0.64 ), equivalent to 10 points (95\% CI 8 to 13 points) on a scale of $0-100$ points. Furthermore, high-quality evidence from 13 studies (1,073 participants) revealed that exercise improved quality of life (SMD $0.28,95 \%$ CI 0.15 to 0.40 ) equivalent to 4 points ( $95 \%$ CI 2 to 5 points) on a scale of 0-100 points.

Encouragingly, Fransen et al. (2015) reported a sustained treatment effect at 2-6 months after intervention. Evidence from 12 studies (1,468 participants) 
indicated reduction in pain (SMD $-0.24,95 \% \mathrm{CI}-0.35$ to -0.14 ), equivalent to 6 (3-9) points on a scale of $0-100$ points. Ten studies (1,279 participants) indicated sustainability of effect on physical function (SMD $-0.15,95 \%$ CI -0.26 to -0.04), equivalent to improvement of $3(1-5)$ points on a scale of 0-100-points. This confirmed the large significant ES (SMD -0.94, 95\% CI -1.31 to -0.57) of exercise on knee pain reported by Tanaka et al. (2013) based on eight studies (unclear number of participants).

In a review comparing exercise with control in 12 studies (808 participants),

Devos-Comby et al. (2006) concluded that exercise had only a small-to-moderate effect on physical outcomes as assessed by self-report and direct measures with ES $=0.29$ (95\% CI 0.23 to 0.36); a small positive effect was seen on direct measures of impairment in 11 studies (740 participants) with a mean ES $=0.15$ (95\% CI 0.08 to 0.23 ). This is supported by the findings of two descriptive reviews (Focht, 2006; Petrella, 2000). Focht (2006) reported significant differences in pain between exercise and control interventions with small (0.25) to large (2.75) ESs based on 18 studies (2,320 participants). Petrella (2000) found small-tomoderate beneficial effects of exercise treatment for pain, small beneficial effects on disability, and moderate-to-great beneficial effects were observed according to patient global assessment of effect.

In a review of 12 studies (unknown number of participants) comparing three types of exercise interventions, Jansen et al. (2011) reported that the ES on pain for strength training was ES $=0.38$ (95\% CI 0.23 to 0.54), for exercise, it was ES $=0.34$ (95\% CI 0.19 to 0.49 ), and the ES on pain for exercise plus manual mobilization was ES = 0.69 (95\% CI 0.42 to 0.96). Furthermore, each intervention also improved physical function significantly. However, metaregression indicated that exercise plus manual mobilizations improved pain significantly more than exercise alone ( $p=0.03$ ). Subgroup analyses by Tanaka et al. (2013) showed a larger effect for non-weight-bearing strengthening exercise (SMD -1.42, 95\% CI -2.09 to -0.75 ) compared with weight-bearing strengthening exercise (SMD -0.70, 95\% CI -1.05 to -0.35 ) and aerobic exercise (SMD $-0.45,95 \%$ CI -0.77 to -0.13 ).

Juhl et al. (2014) examined the effects of different types of exercise programs on pain in 47 studies (4,028 participants). Positive effects on pain relief were found for aerobic, resistance, and performance exercise (SMD 0.67, 0.62, and 0.48 , respectively; $p=0.733$ ). Single-type exercise programs were more efficacious than programs that included different exercise types (SMD 0.61 versus $0.16 ; p<0.001$ ). The effect of aerobic exercise on pain relief increased with an increased number of supervised sessions (slope 0.022, 95\% CI 0.002 to 0.043). More pain reduction occurred with quadriceps-specific exercise than with general lower limb exercise (SMD 0.85 versus $0.39 ; p=0.005$ ) and when super41 
$p=0.017)$. No impact of intensity, duration of individual sessions, or patient characteristics was found. Similar results were found by Roddy et al. (2005) in 13 studies. Pooled ESs for pain were 0.52 for aerobic walking and 0.39 for quadriceps strengthening, and for self-reported disability, ES was 0.46 for aerobic walking and 0.32 for quadriceps strengthening.

\section{Exercise for OA of the Lower Limb}

Ten reviews included studies involving participants with OA of either their hip or knee (Bartels et al., 2007; Brosseau et al., 2004; Daly Iversen, 2010; Pelland et al., 2004; Pisters et al., 2007; Tiffreau et al., 2007; Uthman et al., 2014; van Baar, Assendelft, Dekker, Oostendorp, \& Bijlsma, 1999; Waller et al., 2014; Wallis \& Taylor, 2011)

Uthman et al. ( 2014) in an analysis of 60 studies (44 knee, two hip, 14 mixed with 2,118 participants) reported that for pain relief, several types of exercise programs were significantly more effective than control. These included (a) strengthening, (b) flexibility plus strengthening, (c) flexibility plus strengthening plus aerobic, (d) aquatic strengthening, and (e) aquatic strengthening plus flexibility. Furthermore, a combined intervention of strengthening, flexibility, and aerobic exercise was also significantly more effective than the no-exercise control for improving limitation in function (SMD -0.63, 95\% credible interval -1.16 to -0.10 )

ESs from 11 studies, 10 on the knee and 1 on the hip (van Baar et al., 1999) indicated small-to-moderate beneficial effects of exercise therapy on pain, small beneficial effects on both disability outcome measures, and moderate-to-great beneficial effects according to the patient's global assessment of effect. However, only two studies had an acceptable validity score as well as sufficient power and another two studies had an acceptable validity score, but low power.

In a best-evidence synthesis of 11 studies ( 5 high and 6 low quality) (Pisters et al., 2007), strong evidence was found for no long-term effectiveness on pain and self-reported physical function, moderate evidence for long-term effectiveness on patient global assessment of effectiveness, and conflicting evidence for observed physical function. For exercise programs with additional booster sessions, moderate evidence was found for long-term effectiveness on pain, selfreported physical function, and observed physical function.

This is further confirmed by descriptive reviews of 12 studies (1,363 participants) (Brosseau et al., 2004) and 18 studies (1 hip, 6 hip and knee, 11 knee) (Daly Iversen, 2010) that indicated that various forms of aerobic exercise, such as a walking program, jogging in water, yoga, and tai chi can have statistically significant effects on pain, joint tenderness, functional status, and respiratory capacity for patients with OA. 
A review of 22 studies (2,325 participants) concluded that strengthening exercises alone have some effects on improving pain and functional outcomes (Pelland et al., 2004). However, in order to maximize their effectiveness, it is necessary to combine them with a more complete exercise program including range of motion (ROM), stretching, functional balance, and aerobic exercises.

\section{Individual Versus Group Exercise}

The effectiveness of individual or collective group exercise programs for knee or hip OA was examined in 29 studies (21 knee, 1 hip, and 7 knee and hip) (Tiffreau et al., 2007). The authors reported that the benefit of individual and group exercise programs is low-to-moderate for pain, strength, and ability to walk, and that there is no evidence of the superiority of one modality over the other. However, they observed that effectiveness is not maintained over time if the exercise program is not continued.

\section{Aquatic Versus Land-Based Exercise} The effects of aquatic based exercise were examined by Waller et al. (2014). They included 11 studies (6 knee and hip, 3 knee only, 1 hip only, and 1 any lower limb OA in a total of 1,092 participants). Effects directly after intervention revealed a small but significant effect from aquatic exercise on pain (SMD 0.26, 95\% CI 0.11 to 0.41), stiffness (SMD 0.20, 95\% CI 0.03 to 0.36), self-reported and objectively measured physical functioning (SMD 0.30, 95\% CI 0.18 to 0.43; and SMD $0.22,95 \%$ CI 0.07 to 0.38 , respectively), physical functioning at activity level (SMD 0.22, 95\% CI 0.01 to 0.42), ROM (SMD 0.56, 95\% CI 0.14 to 0.99), and quality of life (SMD $0.24,95 \%$ CI 0.04 to 0.45 ). Only two studies examined effects at 3- and 6-month follow-up, but the effects had been lost at both follow-up measurement points.

An earlier meta-analysis comparing aquatic with land-based exercise at the end of treatment for combined knee and hip OA revealed that there was a small-to-moderate effect on function (SMD 0.26, 95\% CI 0.11 to 0.42 ) and a small-to-moderate effect on quality of life (SMD 0.32, 95\% CI 0.03 to 0.61). A minor effect of a $3 \%$ absolute reduction ( 0.6 fewer points on a $0-20$ scale) and $6.6 \%$ relative reduction from baseline was found for pain. There was no evidence of effect on walking ability or stiffness immediately after the end of treatment (Bartels et al., 2007). From the one trial including participants with hip OA alone, which compared aquatic exercise with land-based exercise, no evidence of effect on pain, function, or quality of life was observed. However, for the one study including participants with knee OA, there was a large effect on pain (SMD 0.86, $95 \%$ CI 0.25 to $1.47 ; 22 \%$ relative percent improvement), immediately after the 
Positive effects of exercise programs involving aquatic strengthening and aquatic strengthening plus flexibility on pain were also confirmed by Uthman et al. (2014).

\section{Exercise Prior to Surgery}

Only one review (Wallis \& Taylor, 2011) has examined the effects of exercise prior to joint replacement surgery. They included 23 studies (1,461 participants) and found moderate-quality evidence that preoperative exercise interventions for knee OA reduced pain prior to knee replacement surgery (SMD 0.43, 95\% CI 0.13 to 0.73 ). Meta-analyses provided evidence of low-to-moderate quality that exercise interventions for hip OA reduced pain (SMD 0.52, 95\% CI 0.04 to 1.01 ) and improved activity (SMD $0.47,95 \%$ CI 0.11 to 0.83 ) prior to hip replacement surgery. Meta-analyses provided low-quality evidence that exercise with education programs improved activity after hip replacement with reduced time to reach functional milestones during hospital stay, for example (SMD 0.50, 95\% CI 0.10 to 0.90), for first-day walking.

Is Exercise Safe?

Adverse events attributed to exercise programs have been reported but these are typically small numbers ( Fransen et al., 2014; Fransen et al., 2015; Waller et al., 2014), and were all related to increased pain and specifically in the knee or back (Fransen et al., 2015).

\section{Considerations}

There are many common deficiencies in the reports of the primary studies included in the reviews considered in this chapter, many of which are detailed in the extension to the Consolidated Standards of Reporting Trials (CONSORT) statement for randomized controlled trials of nonpharmacological interventions

Many studies do not include sufficient detail to allow subsequent com-

Other areas of consideration include level of supervision if any, type of 
Blinding is also a particular issue for exercise interventions but not impossible, and authors should explain how they attempted to blind participants and therapists. This is of course closely related to sham therapy, again difficult but not impossible to achieve for exercise interventions. This could, for example, include the use of generalized stretching programs and attention only activities.

The choice of appropriate, valid, and reliable outcome measures is an area that should receive attention. The conclusions of a recent systematic review suggest that further good-quality research investigating measurement properties of performance (function) measures including responsiveness and interpretability in people with hip and/or knee OA is needed (Dobson et al., 2012).

Other issues relate to the duration of surveillance. This is often inadequate; typically, data ends on completion of the intervention with no follow-up or at best short-term follow-up data. It has been demonstrated that initial compliance with exercise programs is high because of loyalty to the therapist (Campbell et al., 2001). However, reasons underpinning continued compliance is more complex, involving willingness and ability to accommodate exercises within everyday life, the perceived severity of symptoms, attitudes toward arthritis and comorbidity, and previous experiences of OA. A necessary precondition for continued compliance was the perception that the exercise was effective in ameliorating unpleasant symptoms. A Cochrane review (Jordan, Holden, Mason, \& Foster, 2010) concluded that interventions such as supervised or individualized exercise therapy and self-management techniques might enhance exercise adherence for chronic musculoskeletal pain in adults.

Many studies of exercise lack power; the sample size should be adequate to provide a high probability of detecting as significant an ES of a given magnitude if such an effect actually exists. Furthermore, the severity of OA of participants is frequently not quantified by the authors of studies. This could have important implications on the data obtained and conclusions drawn from these studies. Future studies should include this information, as this would allow future reviews to undertake subgroup analysis in which the intervention effect is evaluated in a defined subset of the participants by severity of symptoms. Finally, adverse event reporting is often inadequate; this can mislead by introducing a bias favoring the intervention. The emphasis on treatment benefit, together with lack of information on harmful effects, creates difficulties for people who are trying to make balanced decisions (Cochrane Adverse Effects Methods Group, 2015).

\section{CONCLUSIONS}

This chapter has summarized the findings of recent systematic reviews that have examined the effectiveness of exercise on pain and function compared with 
controls: land- versus water-based exercise, individual versus group exercise, and exercise prior to surgery for people with OA of the hip, the knee, and lower limb (hip and knee combined). Generally, most forms of exercise have been demonstrated to have significant positive effects on pain and function at the end of the exercise program, but unless participants continue the program, the sustainability of these effects in the longer term is questionable. However, exercise appears to be a safe intervention with few adverse effects reported.

There are a number of important considerations relating to the design and reporting of primary research in this area. Future studies are needed to determine the optimum dose of exercise programs (intensity, duration, and frequency) and to determine methods to optimize compliance with the aim of integrating exercise into people's lifestyle. It is important, however, that any further studies should be cognizant of the reporting guidelines of the CONSORT statement.

\section{REFERENCES}

Bartels, E. M., Lund, H., Hagen, K. B., Dagfinrud, H., Christensen, R., \& DanneskioldSamsøe, B. (2007). Aquatic exercise for the treatment of knee and hip osteoarthritis. Cochrane Database of Systematic Reviews, (4), CD005523. doi:10.1002/14651858. CD005523.pub2

Bellamy, N., Kirwan, J., Boers, M., Brooks, P., Strand, V., Tugwell, P., et al. (1997). Recommendations for a core set of outcome measures for future phase III clinical trials in knee, hip, and hand osteoarthritis. Consensus development at OMERACT III. The Journal of Rheumatology, 24(4), 799-802.

Boutron, I., Moher, D., Altman, D. G., Schulz, K., Ravaud, P., \& CONSORT group. (2008). Extending the CONSORT Statement to randomized trials of nonpharmacologic treatment: Explanation and elaboration. Annals of Internal Medicine, 148(4), 295-309.

Brosseau, L., Pelland, L., Wells, G., Macleay, L., Lamothe, C., Michaud, G., et al. (2004). 28 Efficacy of aerobic exercises for osteoarthritis (part II): A meta-analysis. Physical 29 Therapy Reviews, 9(3), 125-145.

Campbell, R., Evans, M., Tucker, M., Quilty, B., Dieppe, P., \& Donovan, J. L. (2001). Why don't patients do their exercises? Understanding non-compliance with physiotherapy in patients with osteoarthritis of the knee. Journal of Epidemiology Community Health, 55(2), 132-138. doi:10.1136/jech.55.2.132

Caspersen, C. J., Powell, K. E., \& Christenson, G. M. (1985). Physical activity, exercise, and physical fitness: Definitions and distinctions for health-related research. Public Health Reports, 100(2), 126-131.

Cochrane Adverse Effects Methods Group. (2015). More about us. Retrieved from http://

Daly Iversen, M. (2010). Managing hip and knee osteoarthritis with exercise: What is the best prescription? Therapeutic Advances in Musculoskeletal Disease, 2(5), 279-290. 
Devos-Comby, L., Cronan, T., \& Roesch, S. (2006). Do exercise and self-management interventions benefit patients with osteoarthritis of the knee? A metaanalytic review. The Journal of Rheumatology, 33, 744-756.

Dobson, F., Hinman, R. S., Hall, M., Terwee, C. B., Roos, E. M., \& Bennell, K. L. (2012). Measurement properties of performance-based measures to assess physical function in hip and knee osteoarthritis: A systematic review. Osteoarthritis Cartilage, 20(12), 1548-1562. doi:10.1016/j.joca.2012.08.015

Focht, B. C. (2006). Effectiveness of exercise interventions in reducing pain symptoms among older adults with knee osteoarthritis: A review. Journal of Aging and Physical Activity, 14(2), 212-235.

Fransen, M., McConnell, S., Harmer, A., Van der Esch, M., Simic, M., \& Bennell Kim, L. (2015). Exercise for osteoarthritis of the knee. Cochrane Database of Systematic Reviews, (1), CD004376. doi:10.1002/14651858.CD004376.pub3

Fransen, M., McConnell, S., Hernandez-Molina, G., \& Reichenbach, S. (2014). Exercise for osteoarthritis of the hip. Cochrane Database of Systematic Reviews, (4), CD007912. doi:10.1002/14651858.CD007912.pub2

Hernández-Molina, G., Reichenbach, S., Zhang, B., Lavalley, M., \& Felson, D. (2008). Effect of therapeutic exercise for hip osteoarthritis pain: Results of a meta-analysis. Arthritis and Rheumatism, 59(9), 1221-1228.

Hootman, J., \& Helmick, C. (2006). Projections of US prevalence of arthritis and associated activity limitations. Arthritis and Rheumatism, 54, 226-229.

Hootman, J., Macera, C., Ham, S., Helmick, C., \& Sniezek, J. (2003). Physical activity levels among the general US adult population and in adults with and without arthritis. Arthritis Rheumatism, 49, 129-135.

Howe, T. E., Rochester, L., Neil, F., Skelton, D. A., \& Ballinger, C. (2011). Exercise for improving balance in older people. Cochrane Database of Systematic Reviews, (11), CD004963. doi: 10.1002/14651858.CD004963.pub3

Howe, T. E., Shea, B., Dawson, L. J., Downie, F., Murray, A., Ross, C., et al. (2011). Exercise for preventing and treating osteoporosis in postmenopausal women. Cochrane Database of Systematic Reviews, (7), CD000333. doi: 10.1002/14651858. CD000333.pub2

Iwamoto, J., Sato, Y., Takeda, T., \& Matsumoto, H. (2011). Effectiveness of exercise for osteoarthritis of the knee: A review of the literature. World Journal of Orthopedics, 2(5), 37-42. doi:10.5312/wjo.v2.i5.37

Jamtvedt, G., Dahm, K. T., Christie, A., Moe, R. H., Haavardsholm, E., Holm, I., \& Hagen, K. B. (2008). Physical therapy interventions for patients with osteoarthritis of the knee: An overview of systematic reviews. Physical Therapy, 88(1), 123-136.

Jansen, M. J., Viechtbauer, W., Lenssen, A. F., Hendriks, E. J. M., \& de Bie, R. A. (2011). Strength training alone, exercise therapy alone, and exercise therapy with passive manual mobilization each reduce pain and disability in people with knee osteoarthritis: A systematic review. Journal of Physiotherapy, 57(1), 11-20. doi: http:// dx.doi.org/10.1016/S1836-9553(11)70002-9 
Jordan, J., Holden, M A., Mason, E., \& Foster, N. (2010). Interventions to improve adherence to exercise for chronic musculoskeletal pain in adults. Cochrane Database of Systematic Reviews, (1), CD005956. doi:10.1002/14651858.CD005956.pub2

Juhl, C., Christensen, R., Roos, E., Zhang, W., \& Lund, H. (2014). Impact of exercise type and dose on pain and disability in knee osteoarthritis: A systematic review and meta-regression analysis of randomized controlled trials. Arthritis and Rheumatology, 66(3), 622-636. doi:10.1002/art.38290

March, L., Smith, E. U., Hoy, D. G., Cross, M. J., Sanchez-Riera, L., Blyth, F, et al. (2014). Burden of disability due to musculoskeletal (MSK) disorders. Best Practice E Research.Clinical Rheumatology, 28(3), 353-366. doi:10.1016/j.berh.2014.08 .002

McAlindon, T., Bannuru, R., Sullivan, M., Arden, N., Berenbaum, F., Bierma-Zeinstra, S., et al. (2014). OARSI guidelines for the non-surgical management of knee osteoarthritis. Osteoarthritis and Cartilage, 22(3), 363-388. doi:10.1016/j.joca .2014 .01 .003

Murphy, L. B., Helmick, C. G., Schwartz, T. A., Renner, J. B., Tudor, G., Koch, G., et al. (2010). One in four people may develop symptomatic hip osteoarthritis in his or her lifetime. Osteoarthritis and Cartilage, 18(11), 1372-1379.

Murphy, L. B., Schwartz, T. A., Helmick, C. G., Renner, J. B., Tudor, G., Koch, G., et al. (2008). Lifetime risk of symptomatic knee osteoarthritis. Arthritis and Rheumatism, 59, 1207-1213.

Paskins, Z., Sanders, T., \& Hassell, A. (2014). Comparison of patient experiences of the osteoarthritis consultation with GP attitudes and beliefs to OA: A narrative review. BMC Family Practice, 15, 46.

Pelland, L., Brosseau, L., Wells, G., MacLeay, L., Lambert, J., Lamothe, C., et al. Tugwell, P. (2004). Efficacy of strengthening exercises for osteoarthritis (part 1): A metaanalysis. Physical Therapy Reviews, 9(2), 77-108.

Petrella, R. J. (2000). Is exercise effective treatment for osteoarthritis of the knee? British Journal of Sports Medicine, 34(5), 326-331. doi:10.1136/bjsm.34.5.326

Pisters, M. F., Veenhof, C., Meeteren, N. L., Ostelo, R. W., Bakker, D. H., Schellevis, F. G., \& Dekker, J. (2007). Long-term effectiveness of exercise therapy in patients with osteoarthritis of the hip or knee: A systematic review. Arthritis and Rheumatism, 57(7), 1245-1253.

Roddy, E., Zhang, W., \& Doherty, M. (2005). Aerobic walking or strengthening exercise for osteoarthritis of the knee: A systematic review. Annals of the Rheumatic Diseases, 64(4), 544-548.

Roddy, E., Zhang, W., Doherty, M., Arden, N. K., Barlow, J., Birrell, F., et al. (2006). Evidence-based clinical guidelines: A new system to better determine true strength of recommendation. Journal of Evaluation in Clinical Practice, 12(3), 347-352.

Romeo, A., Parazza, S., Boschi, M., Nava, T., \& Vanti, C. (2013). Manual therapy and therapeutic exercise in the treatment of osteoarthritis of the hip: A systematic review. Reumatismo, 65(2), 63-74. doi:10.4081/reumatismo.2013.63 
Tanaka, R., Ozawa, J., Kito, N., \& Moriyama, H. (2013). Efficacy of strengthening or aerobic exercise on pain relief in people with knee osteoarthritis: A systematic review and meta-analysis of randomized controlled trials. Clinical Rehabilitation, 27(12), 1059-1071.

Tiffreau, V., Mulleman, D., Coudeyre, E., Lefevre-Colau, M. M., Revel, M., \& Rannou, F. (2007). The value of individual or collective group exercise programs for knee or hip osteoarthritis: Elaboration of French clinical practice guidelines. Annales de Readaptation et de Medecine Physique, 50(9), 741-746.

Uthman, O. A., van der Windt, D. A., Jordan, J. L., Dziedzic, K. S., Healey, E. L., Peat, G. M., \& Foster, N. E. (2014). Exercise for lower limb osteoarthritis: Systematic review incorporating trial sequential analysis and network meta-analysis. British Journal of Sports Medicine, 48(21), 1579.

van Baar, M., Assendelft, W., Dekker, J., Oostendorp, R., \& Bijlsma, J. (1999). Effectiveness of exercise therapy in patients with osteoarthritis of the hip or knee: A systematic review of randomized clinical trials. Arthritis and Rheumatism, 42(7), 1361-1369.

Waller, B., Ogonowska-Slodownik, A., Vitor, M., Lambeck, J., Daly, D., Kujala, U. M., $\&$ Heinonen, A. (2014). Effect of therapeutic aquatic exercise on symptoms and function associated with lower limb osteoarthritis: A systematic review with metaanalysis. Physical Theraphy, 94(10), 1383-1395. doi:10.2522/ptj.20130417

Wallis, J. A., \& Taylor, N. F. (2011). Pre-operative interventions (non-surgical and non-pharmacological) for patients with hip or knee osteoarthritis awaiting joint replacement surgery--a systematic review and meta-analysis. Osteoarthritis and Cartilage / OARS, Osteoarthritis Research Society, 19(12), 1381-1395. doi:10.1016/j. joca.2011.09.001

Walsh, N. E., Mitchell, H. L., Reeves, B. C., \& Hurley, M. V. (2006). Integrated exercise and self-management programmes in osteoarthritis of the hip and knee: A systematic review of effectiveness. Physical Therapy Reviews, 11(4), 289-297.

WHO. (2010a). Global recommendations on physical activity for health. Retrieved from http://apps.who.int/iris/bitstream/10665/44399/1/9789241599979_eng.pdf

WHO. (2010b). International classification of functioning, disability and health (ICF). Retrieved from http://www.who.int/classifications/icf/en/ 


\section{QUERIES:}

AQ1: Please clarify if OMERACT needs spelling out at first instance. Also, please check if the edits in the subsequent sentence are OK.

AQ2: Please check whether the headings and subheadings are identified correctly.

AQ3: Please confirm expansion of OARSI.

AQ4: Please confirm expansion of SMD.

AQ5: The phrase "attention only activities" is not clear and may need rewording.

AQ6: The doi number has been changed to match the details from http://www .ncbi.nlm.nih.gov/pubmed/22944525; is this okay?

AQ7: Please cite the Iwamoto et al. 2011 reference in the text or delete it from the reference list.

AQ8: Please cite the Jamtvedt et al. 2008 reference in the text or delete it from the reference list.

AQ9: Please cite the Walsh et al. 2006 reference in the text or delete it from the reference list.

AQ10: Please confirm that the URL inserted for the WHO 2010a reference is correct. 